\title{
Utilisation appropriée des antihistaminiques
}

\author{
Derek K. Chu MD PhD, Paul Oykhman MD MSc, Gordon L. Sussman MD
}

Citation : CMAJ 2021 April 6;193:E478-9. doi : 10.1503/cmaj.201959-f

Voir la version anglaise de l'article ici : www.cmaj.ca/lookup/doi/10.1503/cmaj.201959

1

Les antihistaminiques sont parmi les médicaments les plus fréquemment et incorrectement utilisés à l'échelle mondiale

Les antihistaminiques sont amplement utilisés pour traiter la rhinoconjonctivite allergique ("rhume des foins») et l'urticaire ${ }^{1-3}$. Évitez les utilisations non indiquées sur l'étiquette pour les troubles où les antihistaminiques sont d'une utilité contestable, comme dans la prise en charge de l'asthme, de l'eczéma, de la toux ou de l'insomnie.

2

Les antihistaminiques de première génération sont associés à des effets indésirables importants et parfois mortels

Les antihistaminiques de première génération (p. ex., la diphénhydramine [Benadryl], la chlorphénamine [ChlorTripolon], l'hydroxyzine [Atarax]) entraînent une sédation, des blessures ainsi que des troubles du sommeil et des fonctions psychomotrices et cognitives, y compris une atteinte aux performances scolaires ${ }^{1-3}$. Une surdose peut entraîner la mort par des effets anticholinergiques et anti-alpha adrénergiques et un allongement de l'intervalle QT (dont les torsades de pointes). Ces antihistaminiques figurent sur la liste de Beer des médicaments potentiellement inappropriés pour les aînés ${ }^{3}$.

Les antihistaminiques plus

récents sont tout aussi abordables et efficaces que les antihistaminiques de première génération en plus d'être plus sécuritaires Des revues systématiques d'essais cliniques randomisés et contrôlés ont démontré que, comparativement aux antihista- miniques de première génération, les antihistaminiques plus récents sont plus sécuritaires (p. ex., $4 \%$ de sédation c. $28 \%)^{2-4}$, ont un effet prolongé (12-24 h c. 4-6 h) et agissent plus rapidement (50 c. $80 \mathrm{~min})^{1}$. En 2013, l'Organisation mondiale de la santé a remplacé la chlorphénamine par la loratadine sur sa liste des médicaments essentiels pour 3 raisons (pour connaître les antihistaminiques privilégiés, consultez le tableau 1 ci-après et l'annexe 1 , accessible en anglais au www.cmaj.ca/lookup/doi/10.1503/cmaj.201959/ tab-related-content) ${ }^{3}$. La bilastine et la fexofénadine sont les options qui présentent le moins d'effets sédatifs. Cependant, aucun antihistaminique ne devrait être consommé avec de l'alcool.

Les antihistaminiques ne devraient pas à être utilisés en remplacement de l'épinéphrine pour traiter l'anaphylaxie

Les antihistaminiques oraux peuvent être utilisés en plus de
Tableau 1 : Résumé des antihistaminiques privilégiés pour les allergies et l'urticaire

\begin{tabular}{|c|c|c|c|c|c|}
\hline \multirow{2}{*}{$\begin{array}{l}\text { Antihistaminiques } \\
\text { (dose unique } \\
\text { standard chez } \\
\text { l'adulte, mg) }\end{array}$} & \multirow{2}{*}{$\begin{array}{l}\text { Disponible } \\
\text { sous forme } \\
\text { liquide? }\end{array}$} & \multirow[b]{2}{*}{$\begin{array}{c}\text { Ordonnance } \\
\text { requise? }\end{array}$} & \multicolumn{2}{|c|}{$\begin{array}{l}\text { Approuvé chez } \\
\text { l'enfant? }\end{array}$} & \multirow{2}{*}{$\begin{array}{l}\text { Données probantes } \\
\text { en matière de } \\
\text { sécurité pendant la } \\
\text { grossesse ou } \\
\text { l'allaitement? }\end{array}$} \\
\hline & & & $\begin{array}{c}\mathrm{SC} \\
\text { (âge) }\end{array}$ & $\begin{array}{l}\text { FDA }^{*} \\
\text { (âge) }\end{array}$ & \\
\hline Bilastine (20) & Non & Oui & S.O. & S.O. & Données lacunaires \\
\hline Cétirizine $(10) \dagger$ & Oui & Non $\ddagger$ & $\begin{array}{l}\text { Oui } \\
\text { (2 ans) }\end{array}$ & $\begin{array}{l}\text { Oui } \\
\text { (6 mois) }\end{array}$ & Oui \\
\hline Desloratadine (5) $\dagger$ & Oui & Non & $\begin{array}{c}\text { Oui } \\
\text { (2 } 2 \text { ans) }\end{array}$ & $\begin{array}{l}\text { Oui } \\
\text { (6 mois) }\end{array}$ & Oui \\
\hline Fexofénadine (180)† & Non & Non & S.O. & S.O. & Oui \\
\hline Loratadine (10)† & Oui & Non $\ddagger$ & $\begin{array}{c}\text { Oui } \\
\text { (2 } 2 \text { ans) }\end{array}$ & $\begin{array}{l}\text { Oui } \\
\text { (1 an) }\end{array}$ & Oui \\
\hline Rupatadine (10) & Oui & Oui & $\begin{array}{l}\text { Oui } \\
\text { (2 ans) }\end{array}$ & S.O. & Données lacunaires \\
\hline
\end{tabular}

Remarque : FDA = Food and Drug Administration, $\mathrm{SC}=$ Santé Canada, S.O. = sans objet.

*FDA ou données probantes d'essais cliniques randomisés et contrôlés pour l'efficacité et la sécurité.

†Plus abordable (consultez l'annexe 1, accessible en anglais au www.cmaj.ca/lookup/doi/10.1503/cmaj.201959/tab-related-content). fOrdonnance non nécessaire, mais peut être recommandée et couvert par des régimes provinciaux comme une voie d'accès abordable à ce médicament. Cependant, les ordonnances sont souvent des obstacles à l'accès ( $p$. ex., les formulaires du Programme d'accès exceptionnel dont les taux d'approbation sont très faibles). 
l'épinéphrine, mais ils ne devraient pas la remplacer pour le traitement de l'anaphylaxie ${ }^{1,3}$. La diphénhydramine administrée sous forme injectable peut causer une vasodilatation et une sédation qui peuvent aggraver l'hypotension et nuire à l'évaluation des patients atteints d'anaphylaxie ${ }^{1,3}$.

\section{L'utilisation de la plupart des antihistaminiques est sécuritaire pendant la grossesse et l'allaitement}

Les revues systématiques d'études observationnelles ne montrent aucune association entre des complications chez le fœtus ou la mère et l'utilisation d'antihistaminiques pendant la grossesse et l'allaitement; ils sont aussi sécuritaires pour les enfants ${ }^{2,3,5}$. Les antihistaminiques les plus récents (comme la rupatadine et la bilastine) ne cumulent pas suffisamment de données probantes pour soutenir leur utilisation au cours de la grossesse.

\section{Références}

1. Fein MN, Fischer DA, O'Keefe AW, et al. CSACl position statement: newer generation $\mathrm{H} 1$-antihistamines are safer than first-generation $\mathrm{H} 1$-antihistamines and should be the first-line antihistamines for the treatment of allergic rhinitis and urticaria. Allergy Asthma Clin Immunol 2019;15:61.

2. Carson S, Lee N, Thakurta S. Drug class review: newer antihistamines: final report update 2. Portland (OR): Oregon Health \& Science University; 2010.

3. Chahal H [for WHO Secretariat]. Section 3: Antiallergics and medicines used in anaphylaxis: histamine-1 receptor antagonists - a critical evaluation to update Section 3. Geneva: The World Health Organization; 2012.

4. Huang CZ, Jiang ZH, Wang J, et al. Antihistamine effects and safety of fexofenadine: a systematic review and meta-analysis of randomized controlled trials. BMC Pharmacol Toxicol 2019;20:72.

5. Gilboa SM, Ailes EC, Pai RP, et al. Antihistamines and birth defects: a systematic review of the literature. Expert Opin Drug Saf 2014;13:1667-98.
Intérêts concurrents : Derek Chu est un chercheur clinicien boursier émergent FCAAI-SCAIC-AllerGen, soutenu par la Fondation canadienne d'allergie, d'asthme et d'immunologie, la Société canadienne d'allergie et d'immunologie clinique et la société AllerGen NCE Inc. (le Réseau allergie, gènes et environnement). Gordon Sussman mentionne des subventions et des honoraires personnels provenant de Novartis, Aralez, CSL Behring, Sanofi, Pediapharm, GSK, Genentech, DBV technologies, Aimmune, AstraZeneca, Stallergenes, Merck, Pfizer, Dyax, Biocryst, Greencross, Kendrion, Shire, Leopharma, Regeneron et mdBriefCase de même que des subventions de Novartis, GSK, Genentech, DBV technologies, Aimmune, CSL Behring, AstraZeneca, Stallergenes, Merck, Pfizer, Dyax, Biocryst, Greencross, Kendrion, Leo Pharma, Regeneron, Sanofi, Blueprint, ALK, Amgen et Cliantha indépendamment des travaux soumis. Aucun autre intérêt concurrent n'a été déclaré.

Cet article a été révisé par des pairs.

Affiliations : Division d'immunologie clinique et d'allergologie, Département de médecine (Chu, Oykhman) et Département des méthodes, des données et de l'incidence de la recherche en santé (Chu), Université McMaster et l'Institut de recherche St. Joe, Hamilton, Ont.; Division d'immunologie clinique et d'allergologie (Sussman), Département de médecine, Université de Toronto, Toronto, Ont.

Propriété intellectuelle du contenu : Il s'agit d'un article en libre accès distribué conformément aux modalités de la licence Creative Commons Attribution (CC BY-NC-ND 4.0), qui permet l'utilisation, la diffusion et la reproduction de tout médium à la condition que la publication originale soit adéquatement citée, que l'utilisation se fasse à des fins non commerciales (c.-à-d., recherche ou éducation) et qu'aucune modification ni adaptation n'y soit apportée. Voir : https://creativecommons.org/licenses/by-nc-nd/4.0/deed.fr.

Correspondance : Derek Chu, chudk@mcmaster.ca 